\title{
La formación de los trabajadores sociales en Chile: ¿un asunto de oferta y demanda?
}

\section{The education of social workers at Chile: a matter of supply and demand?}

\author{
Felipe Andrés SARAVIA CORTÉS \\ Centro de Estudios Regionales y Locales. Departamento de Ciencias Sociales \\ Universidad de Los Lagos (Chile) \\ felipe.saravia.cortes@gmail.com
}

Recibido: 01/09/2014\#

Revisado: 06/11/2014

Aceptado: 23/02/2015

Disponible on line: 10/06/2015

\section{Resumen}

Se aborda la formación de trabajadores sociales con el prisma de la demanda y oferta formativa que genera el sistema de educación superior chileno actualmente. ¿Cuántos trabajadores se forman, y cómo se distribuyen territorialmente? ¿Qué características tienen las instituciones formadoras? en cuanto a acreditación, modalidad en que se imparte la carrera, aranceles, etcétera; ¿Qué características tienen los estudiantes? Se revisa, en particular, la información relativa al género y a los tipos de establecimientos educativos de origen, entre otros. Además, se ahonda en el proceso de inserción laboral, revisando indicadores de empleabilidad y rangos salariales comparándolos con otras carreras, y analizando la "rentabilidad" de estudiar Trabajo Social. Los principales hallazgos manifiestan la relación con el aumento de la oferta de programas formativos en Trabajo Social durante los últimos años, además de la expansión de programas no universitarios, que para el año 2017 podrían generar una titulación mayor que la universitaria, implicando escenarios nuevos para la profesión. Otro hallazgo que resalta interesante es el origen de los estudiantes de Trabajo Social, además de las diversas situaciones, según el territorio, para el ejercicio de la profesión. Por último, se concluye con una serie de desafíos para las Escuelas de Trabajo Social chilenas, resaltando la necesidad de generar una planificación racional de la formación de trabajadores sociales en Chile.

Palabras clave: Trabajo social, mercado laboral, capital humano, oferta educacional, educación superior.

\begin{abstract}
This article addresses the education of social workers from the perspective of demand and training opportunities generated by the Chilean higher education system today. Researching about: how many social workers are graduated and how is their geographic distribution? Which are the characteristics of the training institutions, regarding accreditation, funding models, tuition, etc.? Which are the characteristics of the students concerning information on gender and on educational establishments of origin? It also explores the process of labour market integration, reviewing employability indicators and salary levels compared with other category of graduated, and analyzing the "profitability» of studying Social Work. The main findings show the increased of academic programs of social work in recent years, in addition to the expansion of non-university programs, which by 2017 could generate greater number of diploma in social work than those from university, therefore with new scenarios for the profession. Another interesting finding is the origin of social work students, in addition to various situations for the professional practice, according to the territory. Finally, we conclude with a series of challenges for Chilean Social Work Schools, highlighting the need to generate a rational planning of social workers education in Chile.
\end{abstract}

Keywords: Social work, labor market, human capital, educational offer, higher education.

Referencia normalizada: Saravia Cortés, F. A. (2015): «La formación de los trabajadores sociales en Chile: ¿un asunto de oferta y demanda? Cuadernos de Trabajo Social, 28(1): 69-81.

Sumario: Introducción. 1. Descripción cuantitativa del Trabajo Social chileno. 2. Reflexiones y cuestionamientos finales. 3. Referencias bibliográficas.

\section{Introducción}

El sistema universitario chileno contemporáneo tiene su génesis en las reformas educativas ge- neradas durante la dictadura militar de Pinochet, en la década de 1980, que permitieron el rápido aumento del número de universidades existentes 
en el país, así como «el inicio de procesos de profunda privatización que constituyeron al sistema de educación superior en un mercado cuyas características principales son la ausencia de regulaciones, competencia y la liberalización de la oferta y de la demanda» (Améstica, Gaete y Llinas-Audet, 2014, p. 385). En palabras de la galardonada con el premio nacional de periodismo, María Olivia Mönckeberg (2007), se trata de un mercado extremadamente heterogéneo (incluso caótico) y de escasa transparencia, que Waissblüth $^{1}$ (2010) ha llegado a denominar como un modelo de libertinaje de mercado.

¿Cómo ha afectado este contexto a la formación de los y las trabajadoras sociales en Chile? Es la pregunta general que desarrolla este artículo, mediante una caracterización de la oferta formativa en Trabajo Social, el perfil de sus estudiantes, así como el proceso formativo de estos, y su inserción al mundo laboral.

El análisis propuesto se hace necesario como una forma de reflexionar sobre quiénes son los trabajadores sociales en la actualidad y quiénes lo serán en el futuro; una reflexión hecha desde el sistema formativo, pero con implicancias que deben ser abordadas no solo bajo esta perspectiva, sino también desde la esfera política, con miras a una racionalidad amplia acerca de la formación de trabajadores sociales en Chile.

Se propone que la pregunta incluida en el título de este artículo merece una respuesta no simplista, sino compleja; por un lado, que reconozca el hecho de que el mercado en variadas ocasiones (aunque solo hasta cierto punto) se autorregula de forma eficaz, pero que ello no implica que la formación de capital humano en Chile, y de los trabajadores y trabajadoras sociales en este caso particular, deba dejarse completamente en manos del mercado, ya que de esa forma se pierde la capacidad de contar con una mirada prospectiva y estratégica respecto de los requerimientos para el desarrollo del país. Véase, por ejemplo, la demanda insatisfecha de capital humano calificado en el rubro de la minería en el norte chileno, que tomó por sorpresa al sistema educativo nacional, que no contó con los mecanismos prospectivos requeridos (Rodríguez y Veliz, 2012; Mena, 2013).

La idea de que efectivamente el mercado pueda generar una autorregulación pertinente se enmarca en la lógica liberal según la cual, mediante sus variaciones de oferta y demanda, generará los incentivos para que la Universidad se adecúe a las necesidades del territorio; lo que no necesariamente es así debido a que el financiamiento de las universidades proviene principalmente de las familias de los educandos, son estas quienes deciden finalmente, aunque de forma indirecta, qué carreras se imparten en un determinado territorio, y cuántos profesionales de cada disciplina y especialidad se han de formar, lo que puede dar lugar a que algunas universidades, por ejemplo, no impartan las carreras que el territorio requiere para su desarrollo (presente o futuro), porque no tienen demanda por los potenciales educandos.

Entonces, los requerimientos que las familias de los estudiantes generan al sistema de educación superior no necesariamente condicen con los requerimientos reales del territorio en cuanto a capital humano. En este sentido, la universidad es completamente pertinente con los requerimientos de los consumidores, pero en muchas ocasiones inadecuado para los del territorio.

Esto se ve ejemplificado en el hecho de que, al contrario de lo que ocurre en la mayoría de países de la OCDE, Chile está formando en la actualidad a más profesionales universitarios que técnicos, cuando los requerimientos reales dan cuenta de que la proporción de tipo de capital humano formado debería ser a la inversa (Sociedad del Fomento Fabril, 2012; Idea País, 2013; AIEP y ACTI, 2014)

Probablemente, el fenómeno de una Universidad escasamente alineada con los requerimientos del sistema productivo de su entorno, se debe en gran medida a que el sistema de educación superior chileno funciona basándose en ciertos supuestos, propios del neoliberalismo, que indican que los consumidores toman sus decisiones económicas siempre de forma racional, lo cual ha sido desmitificado por Hodgson (1997), quien propone varios escenarios en que esto no ocurre, y que en el caso de la educación superior tiene que ver con que existe una asimetría de información (y de poder) bastante pronunciada entre ofertante y demandante, además de que es difícilmente comprensible para los postulantes de la primera generación universita-

\footnotetext{
1 Presidente de la Fundación Educación 2020.
} 
ria, la amplia y diversa oferta que se les presenta, con la dificultad adicional de prever cómo evolucionará el mercado laboral en el futuro (Brunner, 2003).

Este escenario constituye el contexto en el que se genera la educación de los y las trabajadoras sociales en la actualidad y que, se propone, podría estar afectándola de forma considerable.

Por otro lado, se propone que una revisión respecto de los tópicos planteados permite explicar en alguna medida el posicionamiento simbólico y de estatus social y económico de los trabajadores y trabajadoras sociales en Chile.

Estos asuntos se desarrollan a partir de una revisión de las bases de datos disponibles en la página web oficial del Ministerio de Educación y que, pese a su fácil accesibilidad, al parecer no han sido utilizadas a la fecha, para sistematizar la oferta formativa en Trabajo Social en Chile, por lo que se estima que este trabajo puede generar un aporte para el mejor entendimiento de la realidad de la disciplina en el país.

\section{Descripción cuantitativa del Trabajo So- cial chileno}

\subsection{La oferta formativa en Trabajo Social en Chile}

Según los datos del Ministerio de Educación, en Chile actualmente existen 188 programas de Trabajo Social ofertados, distribuidos en 41 universidades y 31 ciudades, que en conjunto generan un total de 5.584 vacantes $^{2}$ anuales para estudiar Trabajo Social en el país.

Ahora bien, como se observa en la Tabla 1, la mayoría de los programas ofertados se concentran en las tres grandes ciudades universitarias del país, Santiago, Valparaíso y Concepción, que representan el 53,7 por ciento de la oferta nacional.

Un dato relevante es que del total de programas, solo 27 corresponden a universidades pertenecientes al Consejo de Rectores de las Universidades Chilenas (CRUCH), lo que equivale al 14,3 por ciento del total de programas de Trabajo

\begin{tabular}{|l|r|c|}
\hline \multicolumn{1}{|c|}{ Región } & Programas & Universidades \\
\hline Arica y Parinacota & 6 & 4 \\
Tarapacá & 11 & 5 \\
Antofagasta & 6 & 4 \\
Atacama & 6 & 4 \\
Coquimbo & 11 & 5 \\
Metropolitana & 57 & 23 \\
Valparaíso & 21 & 9 \\
O’Higgins & 5 & 4 \\
Maule & 14 & 6 \\
Bio Bio & 23 & 11 \\
Araucanía & 9 & 6 \\
Los Ríos & 5 & 2 \\
Los Lagos & 12 & 4 \\
Aysén & 1 & 1 \\
Magallanes & 1 & 1 \\
\hline
\end{tabular}

Tabla 1. Cantidad de programas de Trabajo Social y Universidades que imparten dichos programas, según la región.

Fuente: Elaboración propia en base a datos de www.mifuturo.cl

Social ofertados en Chile, y al 14,6 por ciento de las vacantes disponibles a nivel nacional.

Por otro lado, resulta interesante constatar que la oferta total de programas formativos universitarios en Trabajo Social ha aumentado de forma sostenida durante los últimos años. De hecho, entre los años 2010 y 2014 hubo un incremento del 36,2 por ciento, pasando de 138 programas ofertados en 2010, a 188 en 2014, tal y como se aprecia en el Gráfico 1.

Otro asunto relevante para el análisis de la oferta formativa es su calidad, que actualmente se mide principalmente con el proceso de acreditación de las carreras, impulsado por la Comisión Nacional de Acreditación (CNA). Respecto de este punto, las carreras de Trabajo Social presentan cifras poco alentadoras: solo el 26,6 por ciento de los programas ofertados en 2014 se encuentran acreditados. Sin embargo, cuando se analiza por separado la situación de los programas de las universidades del Consejo de Rectores de las Universidades Chilenas, la cifra sube al 44,4 por ciento $^{3}$, lo que indica que estos

${ }^{2}$ Se consideran aquí las vacantes disponibles para ingresar ya sea en el primer o segundo semestre. Las vacantes para ingresar solo durante el primer semestre son 4.434 plazas.

${ }^{3}$ Resulta interesante constatar que, haciendo un filtro aún más preciso, en el caso de la acreditación de las carreras impartidas por las universidades estatales, la cifra es del 36,8 por ciento; menor que la acreditación obtenida por los programas impartidos por las universidades del Consejo de Rectores de las Universidades Chilenas en general. 


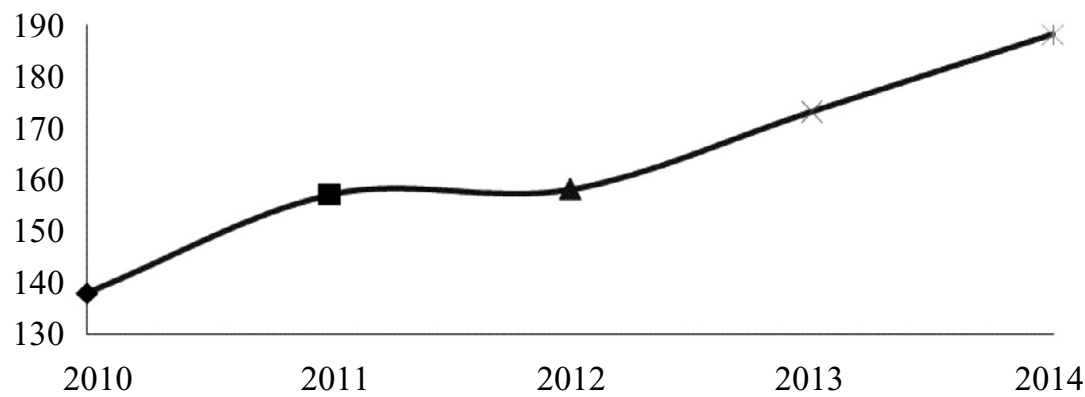

Gráfico 1. Evolución temporal de la oferta de programas formativos universitarios en Trabajo Social en Chile (2010-2014).

Fuente: Elaboración propia en base a datos de www.mifuturo.cl

programas, en general, presentan una mayor calidad que los impartidos por las universidades privadas ${ }^{4}$.

En cuanto a los aranceles, la oferta formativa es bastante diversa. El programa más barato (Universidad Bolivariana) tiene un costo de 730.000 pesos chilenos anuales ${ }^{5}$, en tanto que para el más caro (Pontificia Universidad Católica de Chile) su coste es de 3.325 .000 pesos chilenos (¡4,5 veces más!). Además, en términos generales, es posible encontrar que el monto del arancel es mayor cuando la carrera se encuentra acreditada (Tabla 2).

La acreditación entonces, se constituye en un elemento de marketing institucional que permite aumentar la rentabilidad del «producto» comercializado $\mathrm{y}$, al mismo tiempo, un mecanismo de segmentación socioeconómica del estudiantado, puesto que en términos generales, los «consumidores» tienden a adaptar sus prác-

\begin{tabular}{|c|c|c|}
\hline \multicolumn{1}{|c|}{ Aranceles } & $\begin{array}{c}\text { Programas } \\
\text { acreditados }\end{array}$ & $\begin{array}{c}\text { Programas } \\
\text { no acreditados }\end{array}$ \\
\hline $\begin{array}{l}\text { Arancel promedio } \\
\text { Arancel mediano }\end{array}$ & 1.792 .312 & 1.437 .248 \\
1.795 .500 & 1.386 .500 \\
\hline
\end{tabular}

Tabla 2. Aranceles anuales de programas universitarios de Trabajo Social en Chile según acreditación de calidad (2014) (en pesos chilenos).

Fuente: Elaboración propia en base a datos de www.mifuturo.cl ticas de consumo a sus capacidades de pago, las cuales están asociadas, a su vez, a una determinada posición social, económica, y simbólicocultural previa.

Un último elemento a mencionar, dentro de esta caracterización de la oferta de programas universitarios de Trabajo Social, representa la relación con la modalidad en la que se imparten estos programas. Casi la totalidad de los programas se imparten de forma presencial, aunque destacan tres casos: la Universidad de la República, que cuenta con un programa en Rancagua de modalidad semipresencial, y la Universidad Bolivariana que, desde el 2013, cuenta con dos programas en la modalidad e-learning.

Ahora bien, de los programas presenciales, el 67,6 por ciento se imparte en jornada diurna y el 28,6 por ciento en la modalidad vespertina. La universidad de Aconcagua oferta en varias de sus sedes programas impartidos en una jornada en categoría «otra» (probablemente con clases solo los fines de semana).

\subsection{La matriculación en las carreras de Trabajo Social en Chile}

Durante el año 2013, de acuerdo con las bases de datos del Ministerio de Educación, 13.357 personas se encontraban estudiando Trabajo Social en las universidades chilenas (lo que corresponde al 1,7 por ciento de la matricula total de grado), de las cuales $2.846^{6}$ correspon-

\footnotetext{
${ }^{4}$ La relación entre acreditación y calidad de la docencia puede ser cuestionable. Sin embargo, existen hallazgos a nivel exploratorio que dan cuenta de que, en efecto, existe una relación en este sentido (IPSOS, 2010).

${ }^{5}$ Se trata de un programa especial, de modo vespertino, para funcionarios públicos, que se imparte en Santiago de Chile e Iquique.

${ }^{6}$ Este es un dato aproximado, probablemente bastante menor de la matricula total real, ya que las bases de datos disponibles tienen varias lagunas de información, debido a carreras que no informan de la cantidad de alumnos matriculados.
} 

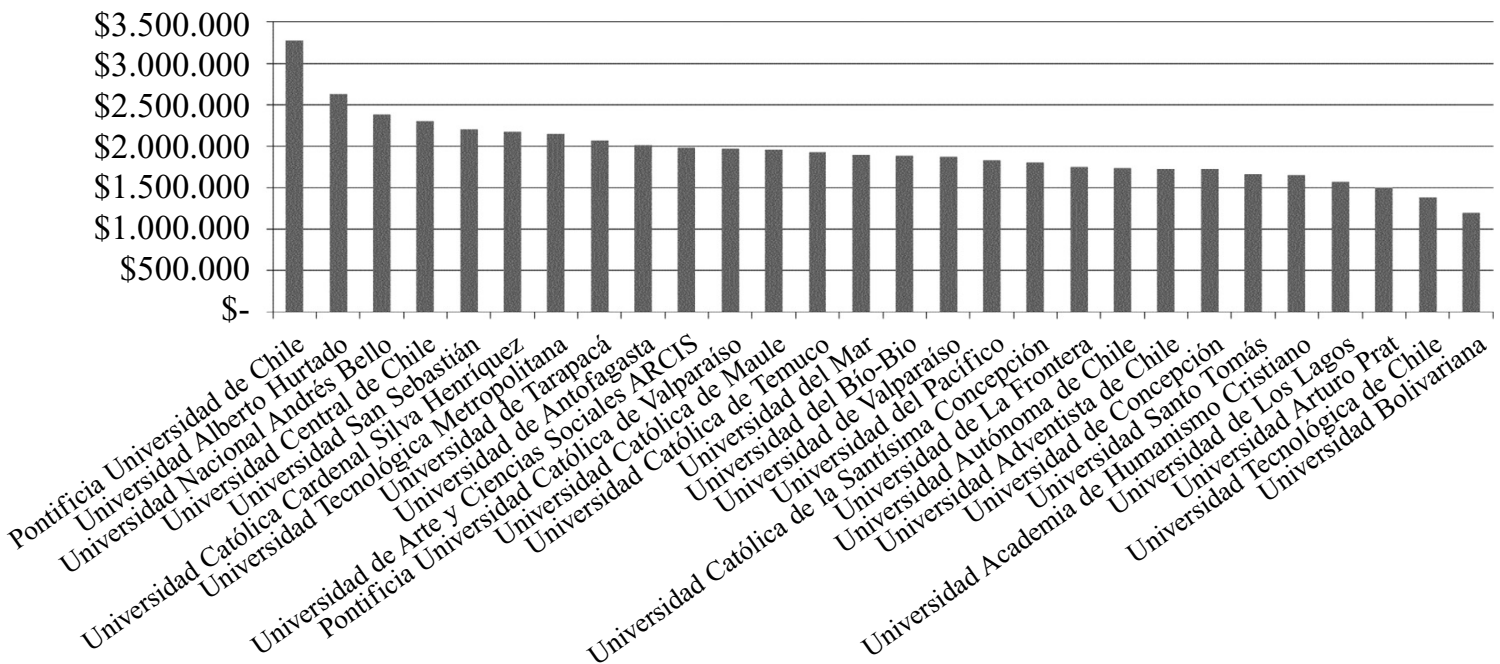

Gráfico 2. Aranceles de las carreras de Trabajo Social en las universidades chilenas (2013).

Fuente: Elaboración propia en base a datos de www.mifuturo.cl

dían a matrículas de primer año. De estas 2.846 personas, el 37,45 por ciento, estudian en la Región Metropolitana, y si se consideran las tres regiones con mayor cantidad de universidades, es decir, la región metropolitana, además de Bío Bío y Valparaíso, concentran el 66,37 por ciento de la matrícula del país, una cifra aún más alta que el 53,7 por ciento de la oferta formativa en Trabajo Social que concentran estas tres regiones.

En cuanto a la evolución temporal de la matriculación, como se observa en el Gráfico 3, en todas las regiones se ha mantenido más o menos estable, a pesar del aumento del 36,2 por ciento de la oferta de programas. No se aprecia un aumento significativo de la matriculación a nivel nacional ni en ninguna de las regiones en particular, lo que contrasta con el incremento de la matriculación que ha experimentado el sistema de educación superior en términos globales. En efecto, desde 2007 a 2013, la matriculación del sistema de educación superior en general se incrementó un 51,6 por ciento.

Otro aspecto interesante de la matriculación en Trabajo Social es que, aunque la proporción de hombres matriculados sigue siendo bastante menor que la de mujeres, entre los años 2007 y 2013 se aprecia un leve aumento de cuatro pun-

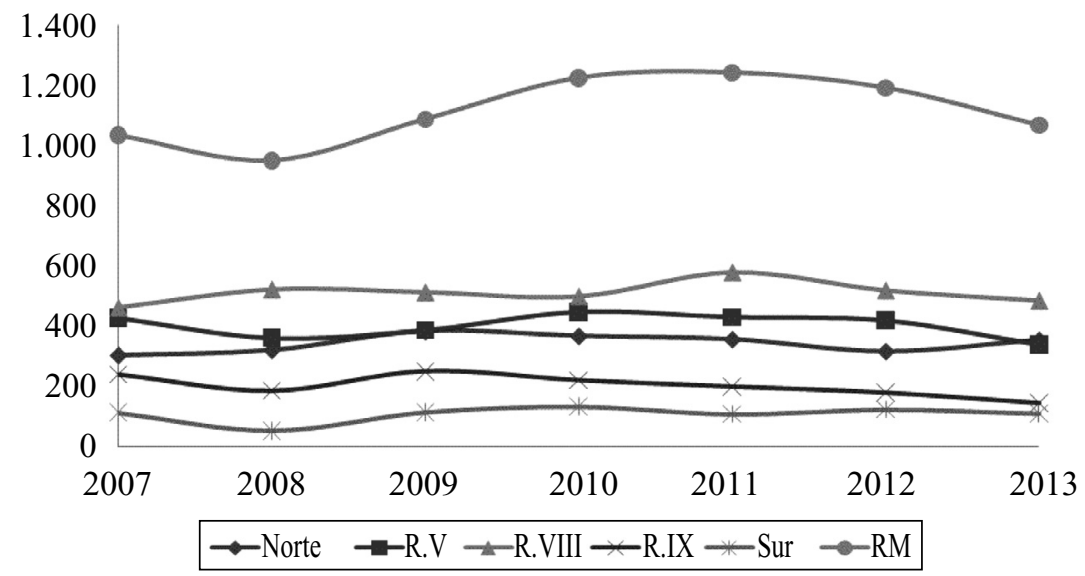

Gráfico 3. Evolución de las matrículas de primer año en Trabajo Social en Chile (2007-2013)*.

* En la elaboración de este gráfico se incluyeron dentro de la macro-región norte a las regiones 1, 2, 3 y 15, en tanto que en la macro-región sur, se incluyeron las regiones 9, 10, 11, 12 y 14.

Fuente: Elaboración propia en base a datos de www.mifuturo.cl 


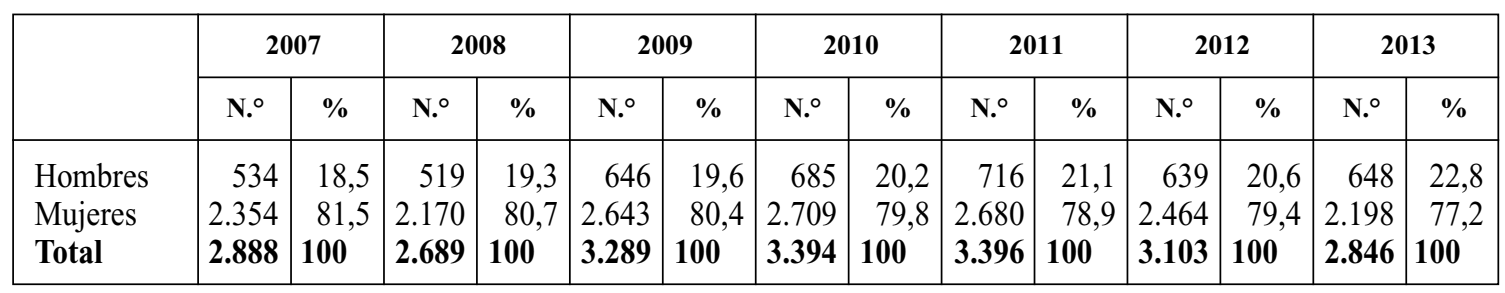

Tabla 3. Proporción de hombres y mujeres matriculados en el primer año de las carreras de Trabajo Social en Chile (2007-2013).

Fuente: Elaboración propia en base a datos de www.mifuturo.cl

tos porcentuales en la representación masculina, tal y como se observa en la Tabla 3.

La sobrerrepresentación femenina en la carrera de Trabajo Social se encuentra probablemente ligada al origen de la disciplina, asociada fundamentalmente a un rol de género, no representando la realidad del sistema universitario en general. En efecto, el sistema de educación superior chileno ha ido incrementando la matriculación femenina durante las últimas tres décadas. En 1984 la representación femenina alcanzaba el 42,6 por ciento, en tanto que en 2013 la presencia femenina en las universidades sobrepasó la masculina con el 51,6 por ciento.

También asociado a la matriculación en Trabajo Social en Chile, un aspecto interesante para analizar es la trayectoria educativa del estudiantado que ingresa a la carrera; se propone que los resultados en materia de empleabilidad e ingresos se encuentran correlacionados con dichas trayectorias educativas, que no solo son educativas sino también sociales, económicas y culturales.

En este sentido, es una constatación interesante el tipo de establecimiento de origen de los estudiantes que, como se sabe, dice mucho también de su nivel socioeconómico, dada la alta segregación escolar nacional.
Se resalta el hecho de que, a nivel nacional, el promedio de las carreras de Trabajo Social presenta una mayor proporción de estudiantes provenientes de establecimientos municipales (37 por ciento) que en las carreras de las ciencias sociales ( 27 por ciento), y el sistema universitario chileno en general (28 por ciento). Así mismo, Trabajo Social a nivel nacional presenta una menor proporción de estudiantes provenientes de colegios particulares de pago ( 5 por ciento), en contraste con carreras de las ciencias sociales ( 22 por ciento) y del sistema universitario en general (19 por ciento).

Como se aprecia en el Gráfico 4, estos indicadores se modifican levemente cuando se desagrega territorialmente la matriculación. En efecto, la Región Metropolitana presenta mayor cantidad de estudiantes provenientes de colegios privados de pago y menor cantidad de estudiantes provenientes de establecimientos municipales (casi un 50 por ciento menos) que los estudiantes del resto de las regiones del país.

Destacan también las carreras impartidas por universidades privadas pertenecientes al Consejo de Rectores de las Universidades Chilenas, que logran atraer una mayor proporción de estudiantes provenientes de colegios particulares de pago. Entre estas, la Pontificia Universidad Ca-

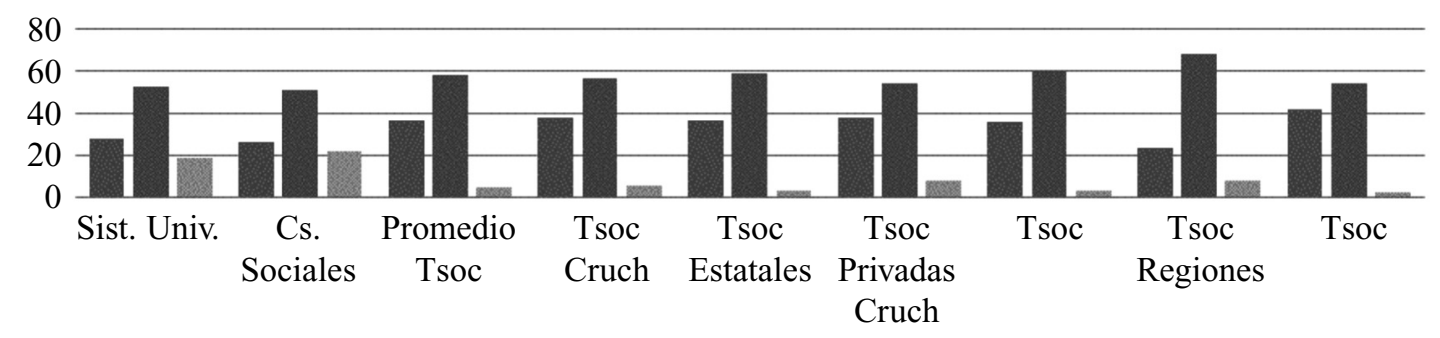

Est. Municipal Est. Subnvenc. 츨 Est. Particular

Gráfico 4. Matriculación de Trabajo Social, según establecimiento educacional de origen, 2013 (porcentaje). Fuente: Elaboración propia en base a datos de www.mifuturo.cl 
tólica de Chile (PUC), con el 27 por ciento, es la que tiene una mayor proporción de estudiantes de este tipo, e incluso logra revertir la distribución observada en las carreras de Trabajo Social y en el sistema universitario chileno en general, en el que la proporción de estudiantes provenientes de establecimientos municipales tiende a ser mayor que la de los colegios privados de pago. Es decir, en el caso de la Pontificia Universidad Católica de Chile, los estudiantes originarios de establecimientos municipales son una minoría, el 21 por ciento.

Las constataciones hechas hasta ahora permiten proponer la idea de que el proceso formativo y sus resultados no son homogéneos en las distintas carreras de Trabajo Social en Chile, no solo porque institucionalmente son distintas, sino porque trabajan con estudiantes de orígenes muy diversos y desiguales.

\subsection{La titulación, la empleabilidad y las re- muneraciones}

La titulación de los trabajadores y trabajadoras sociales, formados en universidades chilenas, fue de 1.865 en 2012, lo que posiciona a la carrera en el décimo lugar de las carreras con mayor cantidad de titulados al año. En efecto, la carrera presenta un crecimiento de la titulación del 8 por ciento respecto de la cantidad de titulados de 2008, tal y como se aprecia en la Tabla 4. Sin embargo, dicho incremento es leve, comparado con el incremento de la titulación de las carreras de Trabajo Social impartidas en Institutos Profesionales y las carreras de Técnico en Trabajo Social o Servicio Social, que en conjunto titulan al año casi la misma cantidad de profesionales y técnicos que titulan las carreras universitarias.

Este es un fenómeno que, como se observa en la Tabla 4, se ha potenciado en los últimos siete años, ya que en 2008 la cantidad de titulados de carreras de Trabajo Social y Servicio Social impartidas por institutos profesionales equivalían solo al 39 por ciento de la titulación generada por las carreras universitarias, llegando en 2012 a un escenario casi de paridad. Es decir, por cada trabajador social universitario titulado, se titula también un técnico en Trabajo Social o asistente social en un instituto profesional.

¿Qué ha provocado tal escenario? ¿Son los requerimientos de la política pública, de los desafíos territoriales los que han impulsado la formación de los trabajadores y trabajadoras sociales (o asistentes sociales) de nivel técnico?; o más bien ¿se trata de un nuevo nicho de mercado encontrado por las instituciones de educación superior chilenas, y que en este momento se está explotando convenientemente?¿Cómo afecta esta realidad a la formación de profesionales dedicados al trabajo o servicio social?, y ¿qué nuevos escenarios generará en el futuro?

Es probable que, si la tendencia se mantiene, en 2017 por cada 2 trabajadores sociales universitarios titulados, se titularán también 3 técnicos en Trabajo Social. Es decir, sin necesidad de una ley por medio, el Trabajo Social chileno actual está volviendo a ser, en un cierto sentido, una carrera predominantemente técnica.

En efecto, lo que naturalmente debía haber comenzando a ocurrir (como ya se ha observado en algunas universidades) era una presión de mercado por generar programas especiales de

\begin{tabular}{|c|c|c|c|c|c|c|c|c|}
\hline \multicolumn{2}{|l|}{ Carreras } & 2008 & 2009 & 2010 & 2011 & 2012 & $\begin{array}{l}\text { \% incremento } \\
\text { (2088-2012) }\end{array}$ & $\begin{array}{l}\text { \% incremento } \\
\text { (2011-2012) }\end{array}$ \\
\hline \multicolumn{2}{|l|}{ Trabajo Social (Ues) } & 1.727 & 1.562 & 1.752 & 1.950 & 1.865 & 8,0 & $-4,4$ \\
\hline \multicolumn{2}{|l|}{ Trabajo Social (IP) } & 419 & 583 & 612 & 782 & 1.023 & 144,2 & 30,8 \\
\hline \multicolumn{2}{|c|}{ Técnico en Servicio Social (IP) } & 255 & 180 & 398 & 502 & 776 & 204,3 & 54,6 \\
\hline Región & \multicolumn{2}{|c|}{ Programas } & \multicolumn{2}{|c|}{ Universidades } & & & & \\
\hline África y Parinacota & \multicolumn{2}{|l|}{6} & \multicolumn{2}{|c|}{4} & & & & \\
\hline Tarapacá & \multicolumn{2}{|l|}{11} & \multicolumn{2}{|c|}{5} & & & & \\
\hline Antofagasta & \multicolumn{2}{|l|}{6} & \multicolumn{2}{|c|}{4} & & & & \\
\hline
\end{tabular}

Tabla 4. Evolución de la titulación de las carreras de Trabajo Social universitaria, Trabajo Social de institutos profesionales y carreras de técnico en Trabajo Social.

Fuente: Elaboración propia en base a datos del Servicio de Información de Educación Superior (SIES 20082012). 
continuidad de estudios, de forma que el creciente número de técnicos en Trabajo Social pudiera optar por el título y el grado académico de trabajadores sociales. Pero conviene preguntarse si una formación de esta naturaleza logra generar las mismas competencias que se desarrollan durante la estancia más larga en la Universidad.

En efecto, en su Declaración de Huilquilemu, la red de Escuelas de Trabajo Social del Consejo de Rectores de las Universidades Chilenas (2014), plantea que:

Los cambios generados, lejos de fortalecer la disciplina, permiten la formación de trabajadores sociales por entidades que por su naturaleza están llamadas a formar a profesionales con un perfil esencialmente técnico y práctico, lo que redunda en una formación con carencias en los aspectos científicos y metodológicos (p. 1).

En lo relativo a la empleabilidad de las carreras de Trabajo Social universitarias, ha sido el 79 por ciento de promedio para los egresados durante su primer año lo que no ha variado significativamente durante la última década ya que, como indican Meller, Lara y Valdés (2009), frente a una «mayor oferta de egresados universitarios en el mercado laboral, la demanda por profesionales universitarios se ha incrementado de manera similar» (p. 8).

En efecto, los autores indican que, en 2001, la empleabilidad del primer año de los egresados de Trabajo Social era también el 79 por ciento, en tanto que en 2006 la cifra bajó al 78 por ciento.

Aunque estas cifras son más bajas que la empleabilidad presentada en 2001 en carreras como: Ingeniera civil en minas (98 por ciento), Odontología (97 por ciento), Ingeniería civil (96 por ciento), Tecnología médica (96 por ciento) o Medicina (94 por ciento), donde casi la totalidad de los egresados cuentan con un trabajo durante su primer año laboral, la empleabilidad de Trabajo Social es bastante superior a la de otras carreras tales como: Arte (58 por ciento en 2001 y 51 por ciento en 2006) o Historia y Letras (67 por ciento en 2001 y 57 por ciento en 2006). Además, puede apreciarse cierta estabilidad en la empleabilidad de los egresados de Trabajo Social que contrasta, por ejemplo, con el brusco descenso de la de los egresados de Sociología entre los años 2001 y 2006: de 91 por ciento a 74 por ciento.

Ahora bien, si se desagrega la situación de los egresados de Trabajo Social, según la Universidad en la que se formaron, aparecen marcadas diferencias. Las tres carreras con peor empleabilidad (Universidad Andrés Bello, Universidad del Pacífico y Universidad Santo Tomás) tienen en común que son universidades privadas que no pertenecen al Consejo de Rectores de las Universidades Chilenas y con múltiples sedes a lo largo del país; en tanto que las carreras con mayor empleabilidad (Universidad de Antofagasta, Universidad Alberto Hurtado y Pontificia Universidad Católica de Valparaíso), son universidades con un mayor arraigo territorial en Antofagasta, Santiago de Chile y Valparaíso correspondientemente.

Pero, ¿qué plantea la diferencia entre una y otra institución en cuanto a empleabilidad? Se propone que algunos de los factores asociados mantienen una relación con el origen socioeconómico y sociocultural de los estudiantes $(\mathrm{Nu}-$ ñez, 2004; Bourdieu y Passeron, 2009, ) y el territorio de origen de los estudiantes (Saravia, 2014), así como el territorio en el que se inserta la Universidad (Améstica, Gaete y Llinas-Audet, 2014)

Podría considerarse también el proceso formativo de la Universidad, incluyendo en este factor indicadores como: la calidad de la docencia y la cantidad de instancias de vinculación con el medio y generación de redes sociales, entre otros; pero por disponibilidad de datos, al menos en este breve estudio, no es posible constatación alguna en este sentido.

Por último, en relación a las remuneraciones de los trabajadores sociales chilenos, es posible encontrar que, como se observa en la Tabla 5, los ingresos promedios de los egresados fluctúan entre 500.000 y 800.000 pesos chilenos mensuales, dependiendo de la Universidad en la que se estudió ${ }^{7}$, siendo el promedio de ingresos mayoritario, el rango entre 600.000 y 700.000 pesos chilenos (54 por ciento de las carreras de Trabajo Social universitarias en Chile).

\footnotetext{
${ }^{7}$ Ello no quiere decir que no existan trabajadores sociales que se salen de la norma y perciben ingresos inferiores al rango normal, así como también bastante por arriba del promedio superior mencionado. Sin embargo, estos no son contemplados en las estadísticas oficiales.
} 
Universidad Nacional Andrés Bello Universidad del Pacífico Universidad Santo Tomás Universidad del Bío-Bio Universidad San Sebastián Universidad de Tarapacá Universidad Autónoma de Chile Universidad Católica de la Santísima... ... Universidad de La Frontera Universidad Tecnolócica de Chile INACAP Universidad Católica Cardenal Silva Henríquez Universidad Católica de Temuco Universidad del Mar Universidad de Los Lagos Universidad de Concepción Promedio Universidad Central de Chile Universidad Bolivariana Universidad Adventista de Chile Universidad Arturo Prat Universidad de Arte y Ciencias Sociales ARCIS Universidad Tecnológica Metropolitana Universidad de Valparaíso

Pontificia Universidad Católica de Chile

Universidad Católica del Maule Universidad Alberto Hurtado

Universidad de Antofagasta

Universidad Academia de Humanismo... Pontificia Universidad Católica de Valparaíso

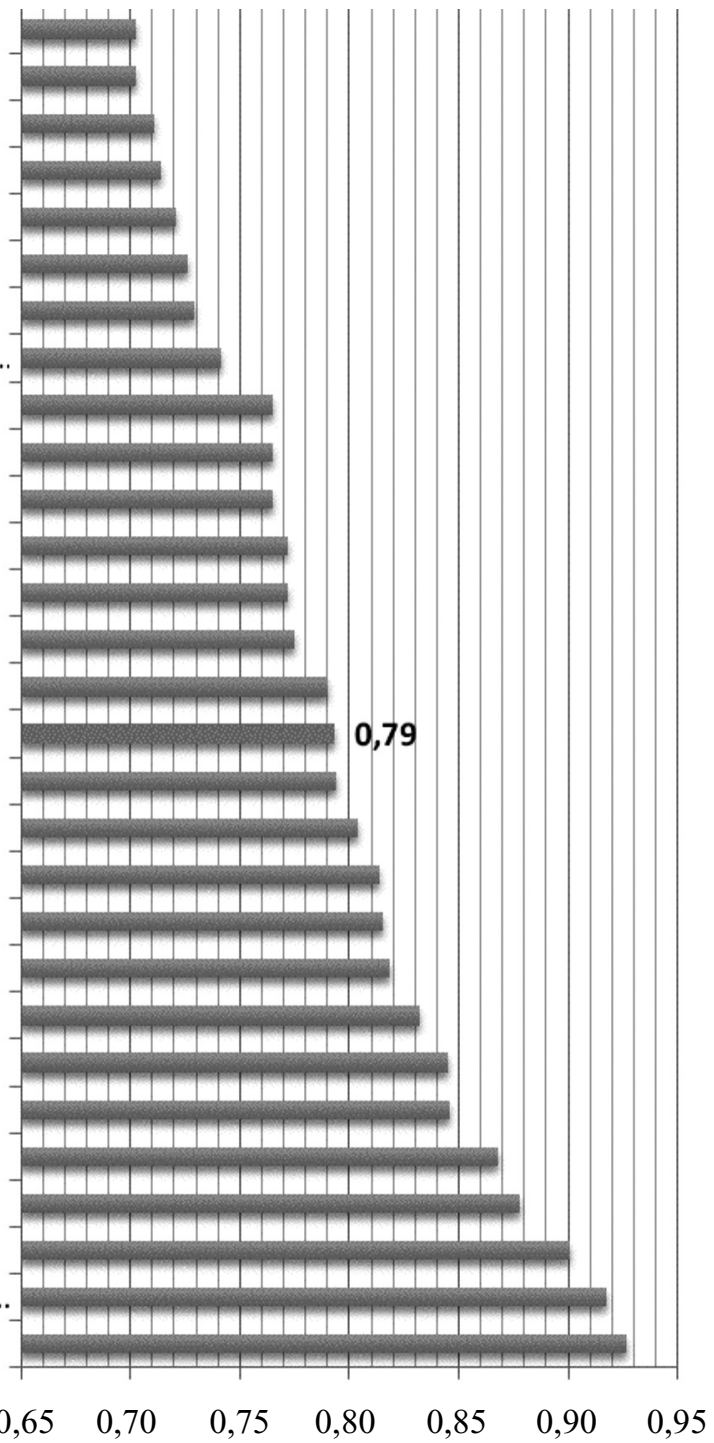

Gráfico 5. Institución según el promedio de «empleabilidad» el primer año de egresados de Trabajo Social (2013).

Como se observa en la Tabla 5, si se compara el nivel de ingresos de los trabajadores sociales con carreras del área de la Administración y Comercio y la Salud, estas aparecen como carreras mucho más rentables que Trabajo Social. Ambas presentan el 46 por ciento aproximado de sus egresados, que perciben ingresos mensuales promedio entre 800.000 y 1.500 .000 pesos chilenos, casi tres veces lo percibido como promedio por los trabajadores sociales en el cuarto año de ejercicio profesional.

Ahora bien, existen también trabajadores sociales egresados de carreras cuyo ingreso promedio al cuarto año se encuentra en el rango en- tre 500.000 y 600.000 pesos chilenos (21 por ciento de las carreras), a la vez que en una proporción minoritaria (11 por ciento), es posible encontrar programas cuyos egresados perciben ingresos promedios ubicados en el rango entre 700.000 y 800.000 pesos chilenos.

¿Qué carreras son las que generan este promedio más alto de la remuneración mensual promedio, y a qué se debe esta diferencia?

Se trata de la Pontificia Universidad Católica de Chile que, como ya se ha mencionado, presenta la mayor proporción de estudiantes provenientes de establecimientos educacionales privados de pago, así como la menor proporción 


\begin{tabular}{|c|c|c|c|c|c|c|c|c|}
\hline \multirow{2}{*}{$\begin{array}{l}\text { Remuneración promedio al cuarto año } \\
\text { (en pesos chilenos) }\end{array}$} & \multicolumn{2}{|c|}{ Trabajo Social } & \multicolumn{2}{|c|}{ Ciencias Sociales } & \multicolumn{2}{|c|}{$\begin{array}{l}\text { Administración } \\
\text { y comercio }\end{array}$} & \multicolumn{2}{|c|}{ Salud } \\
\hline & N. ${ }^{\circ}$ & $\%$ & N. ${ }^{\circ}$ & $\%$ & N. ${ }^{\circ}$ & $\%$ & N. ${ }^{\circ}$ & $\%$ \\
\hline Menos de 500.000 & 0 & 0,0 & 1 & 0,6 & 0 & 0,0 & 2 & 1,3 \\
\hline De 500.000 a 600.000 & 6 & 21,4 & 11 & 7,1 & 2 & 1,7 & 0 & 0,0 \\
\hline De 600.000 a 700.000 & 15 & 53,6 & 32 & 20,8 & 5 & 4,3 & 0 & 0,0 \\
\hline De 700.000 a 800.000 & 3 & 10,7 & 27 & 17,5 & 8 & 7,0 & 6 & 3,9 \\
\hline De 800.000 a 1.000 .000 & 0 & 0,0 & 28 & 18,2 & 24 & 20,9 & 29 & 19,1 \\
\hline De 1.000 .000 a 1.500 .000 & 0 & 0,0 & 5 & 3,2 & 29 & 25,2 & 42 & 27,6 \\
\hline Más de 1.500 .000 & 0 & 0,0 & 0 & 0,0 & 7 & 6,1 & 14 & 9,2 \\
\hline $\mathrm{S} / \mathrm{I}$ & 4 & 14,3 & 50 & 32,5 & 40 & 34,8 & 59 & 38,8 \\
\hline Total & 28 & 100,0 & 154 & 100,0 & 115 & 100,0 & 152 & 100,0 \\
\hline
\end{tabular}

Tabla 5. Programas de Grado de Trabajo Social, Ciencias Sociales, Administración-Comercio y Salud, según el ingreso promedio de sus egresados en el cuarto año (2013).

Fuente: Elaboración propia en base a datos de www.mifuturo.cl

de aquellos que provienen de establecimientos municipales. Además, la Pontificia Universidad Católica de Chile presenta el arancel más elevado del mercado de carreras de Trabajo Social: 3.264.000 pesos chilenos, un 20 por ciento más caro que su predecesora en precio: la carrera impartida por la Universidad Alberto Hurtado (UAH).

Otras carreras con altos promedios de ingresos son: la Universidad Tecnológica Metropolitana y la Universidad de Antofagasta. Ambas tienen aranceles aproximadamente el 40 por ciento más baratos que la carrera impartida por la Pontificia Universidad Católica de Chile, manteniendo los resultados similares e incluso con resultados aún mejores, como es el caso de la Universidad Alberto Hurtado, que presenta una empleabilidad del 90 por ciento al primer año, en contraste con el 84 por ciento de la Pontificia Universidad Católica de Chile.

¿Cuáles son entonces, los factores de mayor peso explicativo en el resultado laboral obtenido por quienes se forman en estas universidades? Sin pretensiones de exhaustividad, se proponen dos: el posicionamiento socioeconómico y sociocultural que los egresados tenían antes del ingreso en la Universidad (medido principalmente, en este caso, mediante el tipo de establecimiento educacional de origen), y el territorio en el que se emplaza la Universidad en la que se formaron.

En efecto, las tres carreras con mejores puntuaciones se encuentran emplazadas en lo que Szary (1996) ha denominado como regiones ga- nadoras, con alto crecimiento económico, y un mercado laboral bullente. De hecho, Meller, Lara y Valdés (2009b), han corroborado este fenómeno, dando cuenta de que el ingreso y la empleabilidad de una misma profesión varían según el territorio. Como se observa en la Tabla 6, la Región del Bío Bío era en los años 2005 y 2006, el peor escenario remunerativo para un trabajador social, en contraposición a la realidad de la zona del norte del país.

Se observa, entonces que, en el caso de la inserción laboral de los trabajadores sociales chilenos, se reproducen las desigualdades territoriales que han sido estudiadas por autores, tales como Aroca (2009), y respecto de las cuales se han desarrollado interesantes propuestas (Von Baer, Torralbo, Saravia y Toloza, 2012; Saravia, 2012).

En este sentido, asistimos a la paradoja de una profesión que se propone luchar contra las desigualdades, pero que al mismo tiempo se ve afectada por ellas.

\section{Reflexiones y cuestionamientos finales}

En este artículo se ha abordado la formación de los trabajadores sociales en Chile, desde la óptica del funcionamiento del mercado educacional y laboral actual, utilizando para ello las bases de datos disponibles en el portal web oficial del Ministerio de Educación.

Los principales hallazgos señalan la relación con el aumento de la oferta de programas formativos en Trabajo Social entre las universidades chilenas; oferta que no ha tenido una corres- 


\begin{tabular}{|l|c|c|c|}
\hline \multicolumn{1}{|c|}{ Regiones } & $\begin{array}{c}\text { Ingreso } \\
\text { medio }\end{array}$ & $\begin{array}{c}\text { Ingreso } \\
\text { mediano }\end{array}$ & $\begin{array}{c}\text { «Empleabilidad» } \\
\text { al primer año }\end{array}$ \\
\hline Zona Norte (Arica y Parinacota, Tarapacá, Antofagasta, & & & \\
y Atacama) & 478.719 & 455.902 & $80 \%$ \\
Región de Valparaíso & 447.483 & 439.832 & $83 \%$ \\
Región Metropolitana & 424.594 & 390.324 & $77 \%$ \\
Región del Bio Bio & 398.905 & 368.080 & $73 \%$ \\
Zona Sur (O'Higgins, Maule, Araucanía, Los Ríos, & & & 813.758 \\
Los Lagos, Aysén, y Magallanes) & 373.945 & $82 \%$ \\
\hline
\end{tabular}

Tabla 6. Ingresos (en pesos chilenos) y «empleabilidad» (en porcentaje) en el primer año de egresados de Trabajo Social (cohortes de egreso, 2005 y 2006P*.

* En la categoría «Norte» se agruparon las regiones 1, 2, 3 y 15; en tanto que en la categoría «Sur» se agruparon las regiones $9,10,11,12$ y 14 .

Fuente: Elaboración propia en base a Meller, Lara y Valdés (2009b).

pondencia en el aumento de la matrícula, cuyo avance ha sido considerablemente más lento.

De hecho, otro hallazgo que debe resaltarse, es la emergencia de las carreras técnicas asociadas a Trabajo Social, que se imparten en los Institutos profesionales, y que ha visto aumentada considerablemente su matrícula en los últimos años, hasta el punto de que si el crecimiento de estas sigue tal y como se ha dado en 2017 , en Chile se estarán titulando 3 técnicos en Trabajo social por cada 2 trabajadores sociales universitarios.

Este fenómeno ya está generando algunos efectos en las carreras de Trabajo Social chilenas, como la presión del mercado por abrir programas de continuidad de estudios, que permitan en un año (y en jornadas flexibles) obtener el grado de licenciado en Trabajo Social para alumnos que se formaron en institutos profesionales.

Así mismo, no extrañaría que la oferta de programas formativos universitarios en Trabajo Social comenzase a disminuir, debido al cierre de carreras por falta de demanda. El fenómeno parece incontrolable, al menos para quienes dirigen las Escuelas de Trabajo Social en las universidades privadas. ¿Existen formas de regular este escenario en universidades del Consejo de Rectores de las Universidades Chilenas? ¿Debiera regularse? Es un debate que este trabajo se propone nutrir.

Por otro lado, aunque quizá no muy novedoso, otro de los hallazgos de la investigación realizada, expresa la relación con los trabajadores sociales que se encuentran en formación actual- mente en Chile. Provienen principalmente de estratos socioeconómicos más bien bajos, y entre sus filas casi no existe presencia de los niveles socioeconómicos (que se encuentran asociados a posiciones socioculturales) más altos de la sociedad chilena ${ }^{8}$ ¿Cómo incide ello en la identidad profesional de los trabajadores sociales en Chile, y de su poder y su incidencia pública? ¿Será que su rol principal de ejecutores de la política social, diseñada principalmente por ingenieros, no es otra cosa sino una reproducción de las desigualdades socioeconómicas y socioculturales de origen? ¿Es posible y deseable hacer algo al respecto?

Por último, ¿qué incidencia tiene las agrupaciones académicas disciplinarias y gremiales en el proceso de definición de la oferta académica en Trabajo Social nacional? ¿Debe dejarse este asunto a las fuerzas del mercado simplemente?

Es cierto que, como plantean Castañeda y Salamé (2010), la carrera ha presentado una buena adaptabilidad a los cambios del mercado, y ha sido capaz de reinventarse a lo largo de su historia, tal y como se ha reflejado en los cambios curriculares a lo largo de casi un siglo de historia profesional, que las autoras sistematizan. ¿Cómo se han ido generando estos cambios? ¿De qué forma las carreras universitarias de Trabajo Social responden o incluso se adelantan a los requerimientos y desafíos que las nuevas problemáticas y demandas sociales generan? Estas y otras preguntas son las que enfrenta en la actualidad el Trabajo Social chileno, y que en este trabajo se propone abrir a discusión.

\footnotetext{
${ }^{8}$ Se deduce de la constatación del tipo de establecimientos educativos de origen de los estudiantes.
} 


\section{Referencias bibliográficas}

AIEP, ACTI. (2014) Escuela de tecnología de la información y telecomunicaciones (TIC): expectativas del profesional del futuro. Chile. Recuperado de: http://www.acti.cl/files/AIEP_ACTI_ESTUDIO_PROFESIONALES_TIC.pdf

Améstica, L., Gaete, H. y Llinas-Audet, X. (2014). Segmentación y clasificación de las universidades en Chile: desventajas de inicio y efectos de las políticas públicas de financiamiento. Ingeniare, 22(3), 384-397. Doi: 10.4067/S0718-33052014000300009.

Aroca, P. (2009). Desigualdades regionales en Chile. Foreign Affairs Latinoamérica, 9(1), 53-63.

Bernasconi, A. (2006). Donde no somos tigres: Problemas de la formación técnica en Chile en el contexto latinoamericano. En foco. Fundación Expansiva, 72,1-24. Recuperado de: http:// 200.111.61.133/tportal/portales/tp4964b0e1bk102/uploadImg/File/EducacionSuperior/4_Problemas_Formacion_Tecnica_Chile.pdf

Bourdieu, P. y Passeron, J.C. (2009). Los herederos. Los estudiantes y la cultura. $2^{\mathrm{a}}$ edición. Buenos Aires: Siglo XXI editores.

Brunner, J.J. (2003). Políticas y mercado de educación superior: necesidades de información. Santiago de Chile: Universidad Adolfo Ibáñez. Recuperado de: http://200.6.99.248/ bru487cl/files/Empleo_capitulo\%25libro.pdf

Castañeda, P. y Salamé, A. (2010) Perspectiva histórica de la formación en Trabajo Social en Chile. Revista electrónica de Trabajo Social. 8,Universidad de Concepción. Disponible en: http:// www.trabajosocialudec.cl/rets/wp-content/uploads/2010/12/historiaformacion. pdf

Comisión Nacional de Acreditación. (2010). Estudio exploratorio sobre efectos de la acreditación institucional en la calidad de la educación superior en Chile. Santiago de Chile: IPSOS. Recuperado de http://www.cnachile.cl/Biblioteca\%20Documentos\%20de \%20Interes/Estudio\%20IPSOS\%20-\%20Informe\%20Final.pdf

Hodgson, G. (1997). The Ubiquity of Habits and Rules. Cambridge Journal of Economics, 21(6), 663-684. Disponible en: http://www.independentinvestor.info/PDF-Downloads/HOW-TO-INVEST-RETURNS-INDEXES-02/doc.1588-\%20Hodgson\% 201997\%20rules\%20of\%20thumb.pdf

Idea País. (2013) Informe educación técnica profesional en Chile. Santiago de Chile. Recuperado de: http://www.ideapais.cl/system/publicacions/archivos/000/000/006/ original/8._T\%C3\%A9cnico_ Profesional.pdf?1380036849

Meller, P., Lara, B. y Valdés, G. (2009a). Comparación intertemporal de ingresos y probabilidad de empleo por carrera al primer año de titulación. Santiago de Chile: Ministerio de Educación de Chile, Departamento de Ingeniería Industrial de la Facultad de Ciencias Físicas y Matemáticas, Universidad de Chile, Centro de Políticas Comparadas de Educación y Universidad Diego Portales. Recuperado de http://www.mifuturo.cl/images/Estudios/Estudios_asociados_a_Futuro_ Laboral/fl003.pdf

Meller, P., Lara, B. y Valdés, G. (2009b). Carreras universitarias (por región): comparación interregional de ingresos al primer año y probabilidad de encontrar empleo. Santiago de Chile: Ministerio de Educación de Chile, Departamento de Ingeniería Industrial de la Facultad de Ciencias Físicas y Matemáticas, Universidad de Chile, Centro de Políticas Comparadas de Educación y Universidad Diego Portales. Recuperado de: http://www.mifuturo.cl/images/Estudios/ Estudios_asociados_a_Futuro_Laboral/fl004.pdf

Mena, A. (2013). Desafios de capital humano en la industria minera. Santiago de Chile: Consejo Minero. Recuperado de: http://www.consejominero.cl/wp-content/ uploads/2013/12/Desaf\%C3\% ADos-de-Capital-Humano-en-la-Industria-Minera. pdf

Mönckeberg, M. (2007). El negocio de las universidades en Chile. Santiago de Chile: Random House Mondadori.

Rodríguez, A. y Véliz, S. (2012). La tardía reacción de las Ues. Qué pasa Minería, 38 Disponible en: http://quepasamineria.cl/index.php/actualidad/item/106-la-tard\%C3\%ADa-reacci $\% \mathrm{C} 3 \%$ B3n-de-las-ues

Nuñez, J. (2004). Discriminación y meritocracia en el mercado laboral en Chile. Economía y Administración 147. Recuperado de http://www.facea.uchile.cl/download.jsp? document $=38364 \&$ property $=$ attachment\&index $=0 \&$ content $=$ 
Red de Escuelas de Trabajo Social del Consejo de Rectores de Universidades de Chile. (2014). Declaración de Hulquilemu. Realizada en el contexto del Congreso Nacional e Internacional, "desafios para la formación en Trabajo Social en contextos de demandas sociales». Recuperado de: http://www.ts.ucr.ac.cr/binarios/declaraciones/decla-0043.pdf

Saravia, F. (2014) Desigualdades entrecruzadas y territorio: propuesta de marco de análisis. Revista electrónica de Trabajo Social, Universidad de Concepción, 11. Disponible en: http://www.revistatsudec.cl/wp-content/uploads/2015/01/Publicaci\%C3\%B3n-Revista-2014.pdf

Saravia, F. (2012) ¿Cómo aumentar stocks de capital humano calificado a nivel local? Políticas públicas comparadas. Revista eletrônica de Programa do Maestrado em Desenvolvimento Regional da Universidade do Contestado, 2(2). Recuperado de: http://www.periodicos.unc.br/index.php/ $\mathrm{drd} /$ article/view/288

Sociedad de Fomento Fabril. (2012). Escasez de 600.000 técnicos se revertiría en 10 años. Recuperado de: http://web.sofofa.cl/noticia/escasez-de-600-mil-tecnicos-profesionales-se-revertiria-endiez-anos/

Szary, A. (1996). Regiones ganadoras y regiones perdedoras en el retorno de la democracia en Chile: poderes locales y desequilibrios territoriales. Revista EURE, 23(70), 59-79.

Von Baer, H., Torralbo, F., Saravia, F. y Toloza, I. (2012) Propuesta de un sistema regional integrado de gestión de capital humano calificado para el desarrollo de las comunas y regiones de Chile. Tercer Encuentro de la Sociedad Chilena de Políticas Pública. Santiago de Chile. Recuperado de: http://www.sociedadpoliticaspublicas.cl/archivos/BLOQUET/Capital_Humano_Regional/ Propuesta $\% 20 \mathrm{de} \% 20 \mathrm{un} \% 20$ Sistema\%20Regional\%20Integrado.pdf

Waissbluth M. (2010). Se acabó el recre.(3 $3^{\mathrm{a}}$ ed.). Santiago de Chile: Random House Mondadori. 